\title{
Tied to expectations: Predicting features speeds processing even under adverse circumstances
}

\author{
Sabine Schwager ${ }^{1} \cdot$ Robert Gaschler $^{2,3}$ • Dennis Rünger ${ }^{4} \cdot$ Peter A. Frensch $^{3,5}$
}

Published online: 20 December 2016

(C) Psychonomic Society, Inc. 2016

\begin{abstract}
When participants predict the upcoming stimulus in a randomized choice reaction task, a match between prediction and stimulus increases processing speed at a level similar to that observed in cueing studies with highly valid cues. This might be taken to suggest that people cannot help but fully use their self-generated, verbalized predictions for preparing task processing. Thus, we tested how flexibly participants can control formation and implementation of predictions. In Experiment 1, we varied validity and response-relevance of predictions. We observed that prediction effects on RT can be boosted by increasing validity, but prevail under adverse circumstances. This was not the case in a control group who read rather than predicted the feature words, suggesting that the effect was specific to predictions as such. Experiment 2
\end{abstract}

Sabine Schwager

sabine.schwager@hu-berlin.de

Robert Gaschler

robert.gaschler@fernuni-hagen.de

Dennis Rünger

dennis.ruenger@gmail.com

Peter A. Frensch

peter.frensch@psychologie.hu-berlin.de

1 Department of Rehabilitation Psychology, Humboldt-Universität Berlin, Georgenstraße 36, 10117 Berlin, Germany

2 Department of Psychology, FernUniversität in Hagen, Universitätsstrasse 33, D-58084 Hagen, Germany

3 Interdisciplinary Laboratory Bild Wissen Gestaltung at Humboldt-Universität, Berlin, Germany

4 Los Angeles, CA, USA

5 Department of Psychology, Rudower Chaussee 18, 12489 Berlin, Germany provided further evidence for limited control of participants over implementing and forming predictions. Participants were provided with practice on stimuli occurring with varying frequency, but neither learned to strategically choose predictions to maximize the number of match trials, nor did they reduce the amount of prediction-based preparation when predicting an infrequent stimulus. As sequential aftereffects of prediction match did not vary with validity, they were identified as an independent effect of verbalizing a response-relevant stimulus feature. The results are consistent with the view that the predicted stimulus feature is represented in the focus of attention in working memory and that the amount of implementation can be subject to weighting.

Keywords Expectations · Predictions · Task preparation . Strategic control

Anticipating characteristics of upcoming situations supports fast and correct goal-directed behavior. This holds for mental tasks as well as overt action. Scientific support for this notion stems from many studies on the effects of providing information in advance to the actual task. Performance can be enhanced, for example, by precueing task sets (e.g., Meiran, 1996; Rogers \& Monsell, 1995) or by incidentally learned regularities that specify some parameters of the upcoming trial in advance, as in sequence learning experiments (e.g., Abrahamse, Jiménez, Verwey, \& Clegg, 2010). In many tasks performance benefits have been attributed to advance activation of stimulus representations before stimulus onset (e.g., Bubic, von Cramon, \& Schubotz, 2010; Dale, Duran, \& Morehead, 2012; Marcus, Karatekin, \& Markiewicz, 2006). Responses are usually faster and more accurate when the situation matches the expected properties compared to when it does not (see Gaschler, Schwager, Umbach, Frensch, \& 
Schubert, 2014, for an overview). This is because expectation can enable the advance preparation of stimulus and response processing (e.g., Mattler, 2005) by preactivation of representations and processes involved in the upcoming task event.

In the current work we ask how flexibly people can control the extent to which self-generated verbalized expectations (i.e., predictions) are used for task preparation. Potentially, internally generated expectations might be coupled to preparatory processes especially tightly. The results of cueing studies have led to the idea that externally suggested expectations do not always lead to preparation. Presumably, cues can be ignored under some circumstances. For instance, Alpay, Goerke, and Stürmer (2009) reported that cues have to be more valid than chance level in order to affect preparatory processes. For predictions, however, there are no studies comparing the impact of different levels of validity. Instead, there are only studies making the case that predictions influence preparation even if they are not valid. Past studies have documented strong behavioral (e.g. Acosta, 1982) and EEG effects of predictions that lacked validity. For instance, Acosta (1982; see also Gaschler et al., 2014, for a recent review) asked participants to predict the upcoming stimulus in a choice reaction task. In this and other studies, participants were faster by about $100 \mathrm{~ms}$ when the predicted stimulus appeared compared to when a different stimulus from the set was shown. This effect occurred, even though stimuli were drawn independently of the predictions (i.e., $50 \%$ probability of match in Acosta, 1982, Exp. 1, with two stimuli), so that the prediction was not valid and matches only occurred by chance. A match between cue and stimulus led to much smaller reaction time advantages over a mismatch (e.g., Acosta, 1982). Furthermore, Acosta reported a substantial reduction in the set size effect in correct predictions as compared to matching cues. Reactions times of stimuli matching the cue were strongly affected by whether two, four, or six stimuli were used in the block. With predictions, set size had only a minor influence.

We argued previously (Gaschler et al., 2014) that forming and verbalizing a prediction has the consequence that the object of thought (i.e., what one is aware of predicting — or aware of not predicting it) is activated in the focus of attention in working memory (e.g., Oberauer, Souza, Druey, \& Gade, 2013). This could explain the lack of a set-size effect reported by Acosta (1982, see above), as well as strong RT benefits for the stimulus that one says one is not expecting (Hacker \& Hinrichs, 1979) or expecting second most (Hacker \& Hinrichs, 1974). It has been argued that the active neural trace implementing the focus of attention in working memory seems to represent just one object at a time (LaRocque, Lewis-Peacock, Drysdale, Oberauer, \& Postle, 2013; LewisPeacock, Drysdale, Oberauer, \& Postle, 2012). This might also imply the following stance: One of the represented objects in working memory seems to have a privileged state.
When a participant is asked to generate and verbalize a prediction, the one stimulus whose representation is strongest on the given trial (potentially by just a small margin over the other candidates) might be picked and exert a strong influence. However, this leaves open the question of whether a stimulus whose representation is leading by a large activation margin when being picked for verbalizing a prediction has a stronger impact on behavior. Studies that show privileged processing of information tied to the object in the focus of attention in working memory (e.g., Oberauer et al. 2013; Schwager \& Hagendorf, 2009) suggest that this is an all-or-none effect. However, Heuer and Schubö (2016) report electrophysiological evidence for flexible weighting of information maintained in visual working memory according to its relevance. Thus, it is not clear whether and how the impact of predictions on preparation depends on validity. According to the intentional weighting principle (e.g., Memelink \& Hommel, 2013), participants might put higher weight on the predictions when experiencing that their predictions are most often met by the stimuli (rather than matching just by chance). However, apart from graded weighting, it is also conceivable that validity impacts the probability with which on a given trial the prediction gains access to the focus of attention in working memory. In this case, an all-or-none effect on the level of individual trials would (by averaging across trials) lead to a larger impact for predictions in a condition with high validity compared to low validity. The intentional weighting principle furthermore would be in line with expectation effects for responseirrelevant features. By making participants verbalize their predictions, even response-irrelevant and invalid predictions might receive some weight. For instance, automatic attentional capture by contents of working memory takes place even when it is not beneficial in a task (Downing, 2000; Pan, 2010). Furthermore, results by Mattler, van der Lugt, and Münte (2006) suggest that expectation mismatch in one aspect of task processing can lead to a cancellation of task preparation based on another expectation (in their case, mismatch of stimulus expectation or response expectation). Therefore, even the match versus mismatch of a response-irrelevant stimulus feature might influence speed in a choice reaction task.

At least for self-generated expectations that do not have to be verbalized, there is evidence that expectation can be ignored versus employed for response preparation depending on task context. A modulation of the behavioral impact of internally generated expectations of perceptual events by usefulness was found in the literature on action-effect anticipation (e.g. Pfister, Kiesel, \& Hoffmann, 2011; Pfister, Kiesel, \& Melcher, 2010). Participants learned what sensorial effect to anticipate even when they did not use anticipation for response preparation and exclusively relied on stimuli for response selection instead. Furthermore, the weight of anticipations versus stimuli might change with practice. While participants used anticipated action effects for response preparation 
when becoming used to a choice reaction task, with practice, they could also more strongly rely on stimulus information, reducing the impact of effect anticipation (e.g., Gaschler \& Nattkemper, 2012; Wolfensteller \& Ruge, 2014). Interestingly, Pfister et al. (2010) showed that usage versus neglect of action effect anticipations generalized across tasks and stimuli and could not flexibly be changed trial by trial. Thus, the literature on anticipation-based action control can be taken to suggest that the impact of predictions can be subject to validity-driven weighting processes, which, however, operate on the time scale of blocks rather than individual trials.

Taken together, it is necessary to explore the conditions that determine the impact of predictions on task processing. In this study, we tested how flexibly participants can control the formation and implementation of their predictions. We therefore manipulated prediction validity and relevance of the predicted stimulus feature. In Experiment 1, relevance was varied by having participants predict either a response-relevant or a response-irrelevant stimulus feature. Validity was manipulated by either meeting predictions in $80 \%$ of the trials or by drawing stimuli independently of the predictions. This was done to probe whether effects of predictions are fixed or can be increased by favorable task conditions. In Experiment 2, in turn, we tested the flexibility of the link between predictions and task preparation by providing participants with practice on a set of stimuli of varying frequency, implying that predictions of frequent stimuli are more likely met than predictions of infrequent stimuli. We asked whether a prediction effect can be obtained even for those predictions met only very rarely or whether participants can flexibly vary the link between preparation and prediction on at trial-by-trial basis, translating only some predictions into preparation.

In both experiments we manipulated circumstances of generating stimulus predictions on a global level over many trials. In addition, we scrutinized the micro level by analyzing the effect of predictions on a trial-by-trial basis. Specifically, we tested whether the influence of a prediction on preparation in the current trial is modulated by prediction match versus mismatch in the preceding trial. Thus, we analyzed the adaptation of prediction effects similar to conflict adaptation demonstrated with the sequential congruency effect (e.g., Gratton, Coles \& Donchin, 1992; see Alpay et al., 2009, for conflict adaptation with cue-induced expectations). If people cannot help but take their predictions seriously, this might also be true for the disconfirmations. In this case, aftereffects of prediction mismatch should be similar for chance-level predictions and for predictions that are most often met by the upcoming stimulus. With a flexible link between prediction and task processing, aftereffects of prediction mismatch might be stronger if predictions matches are frequent. If participants make little use of predictions for task preparation, a disconfirmation should have little impact on the current and subsequent trial.

\section{Experiment 1a}

The first pair of experiments was designed to investigate the influences of prediction validity and degree of action relevance of the prediction on the effectiveness of the prediction in task preparation. We operationalized effectiveness as the prediction (mis)match effect on RT (i.e., the difference between trials in which the stimulus matched and did not match the prediction just verbalized). This difference is referred to as the prediction effect in the following. Three shapes that could occur in one of three colors served as stimuli in a simple threechoice manual reaction task. The response keys were mapped either to the three colors or the three shapes. Before each stimulus presentation, participants were required to verbalize their prediction either concerning color or shape. With this setup it was possible to vary the two experimental factors validity ( $80 \%$ vs. chance) and degree of action relevance (response-relevant vs. irrelevant feature) orthogonally, leading to four between-subjects conditions. We hypothesized that (1) prediction effects are larger, when the prediction is met by the stimulus with a higher probability (high validity $>$ low validity), and (2) that prediction effects are larger, when the prediction is more relevant for task execution - that is, directly relates to response selection (relevant $>$ irrelevant).

\section{Method}

Participants Sixty-two undergraduate students of psychology and other fields ( 38 women, mean age $=24.13$ years, $S D=$ 4.01) participated in individual sessions lasting around $60 \mathrm{mi}-$ nutes. Participants either received partial course credit or were paid $€ 8$ for their time.

Stimuli and design Three different shapes (see Fig. 1) served as stimuli. They were chosen so that each shape could be named by a monosyllabic word in order to provide for approximately equal verbalization times. The shapes were house, cross, and star (in German: Haus, Kreuz, Stern). They were displayed centrally on a light gray background and occupied approximately $22 \mathrm{~mm}$ in diameter (corresponding to a visual angle of about $2.1^{\circ}$ at a viewing distance of $60 \mathrm{~cm}$ ). The stimuli could occur in red, green, or blue. The keys v, b, and $\mathrm{n}$ on a standard keyboard were mapped either to the three shapes or the three colors, depending on which feature was response relevant (response feature).

Stimuli varied independently along the dimensions shape and color. For one feature, participants had to predict the value before stimulus presentation (prediction feature). We manipulated the probability that the feature of the stimulus matched the prediction (validity). This happened either by chance (i.e., on $33 \%$ of the trials), or on $80 \%$ of the trials.

Prediction feature (response-relevant or response irrelevant stimulus feature) and validity were varied between 


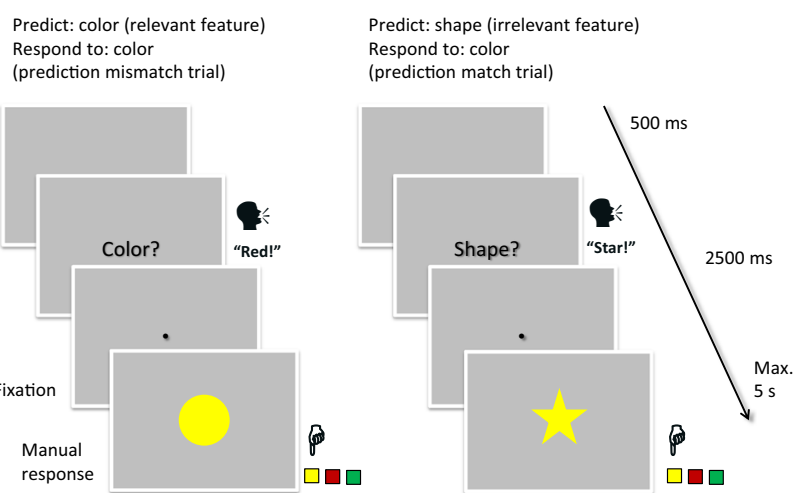

Fig. 1 Example for two trials of the task used in Experiment 1a. On each trial, participants had to verbalize their prediction for the upcoming stimulus (with respect to either its color or its shape). After 2,500 ms, the stimulus appeared on screen, matching or mismatching the predicted feature, and participants had to respond manually by pressing one of three keys. The next trial started $500 \mathrm{~ms}$ after the response. For any given participant, only one of the two stimulus features (shape, color) had to be predicted throughout the task, and only one was response relevant (Color figure online)

participants and stayed the same for a participant over the whole experiment. This resulted in four experimental groups in which the factors response relevance and validity of the predictions are crossed: prediction response relevant but of low validity (relevant-low), prediction response relevant and highly valid (relevant high), prediction response irrelevant and of low validity (irrelevant-low), and prediction response irrelevant but highly valid (irrelevant-high). The specific feature (color and shape) occurred with equal frequencies within these four groups. Response mapping was chosen randomly for each participant.

Procedure The experiment consisted of three short blocks with 20 trials each for instruction and practice and five experimental blocks with 127 trials each. Each trial began with the word Farbe (color) or Form (shape), accompanied by a question mark. This was the signal for the participant to verbalize a prediction on the specified dimension. The prediction feature remained constant over the whole experiment for each participant. The experimenter keyed in the participant's prediction via a second keyboard, and 2,500 ms after its occurrence, the question was replaced by the imperative stimulus, and the participant chose the appropriate response key as fast as possible. The next trial began $500 \mathrm{~ms}$ after the response or after reaching the response deadline of $2,500 \mathrm{~ms}$ with the next question for a prediction.

In the first practice block, participants practiced the manual choice reactions, omitting the verbalization of predictions. In the second practice block, they verbalized a prediction before each stimulus presentation without having to respond manually. In the third practice block, the complete trial procedure was introduced. After the final experimental block, the experimenter asked participants to estimate the proportion of trials in which the stimulus matched the prediction.

\section{Results}

Screening suggested a low error rate, $(M=2.3 \%, S D=1.89)$. Participants were able to fulfil the experimental requirements quite well. On average, only $1 \%(S D=1.21)$ of the trials had to be discarded because of no or too late verbalization.

Predictions The categories (rot, grün, blau, and Stern, Haus, Kreuz - red, green, blue, and star, house, cross) were named with approximately equal frequencies (across conditions the minimum mean frequency was $29.4 \%$ for red in the low-validity/relevant prediction condition, and the maximum mean frequency was $36.0 \%$ for blue in the same condition; $S D$ across conditions $=3.73 \%$ ).

Reaction times and errors Median response times for correct responses and mean error rates were determined for each participant, separately for prediction matches and mismatches. Figure 2 shows the mean of median response times and mean error rates for the four experimental conditions: predictions of the response-relevant stimulus feature in Fig.2a, predictions of the irrelevant feature in Fig. 2b. The figure suggests that prediction effects were larger for highly valid predictions as compared to chance predictions and that prediction effects were more pronounced if the predictions concerned the responserelevant stimulus feature.

To test our hypotheses about validity and relevance as moderators of prediction effects, we subjected median response times and mean error rates to mixed ANOVAs with prediction match (match vs. mismatch) as within-subject factor and the validity conditions (chance and $80 \%$ match) as well as the relevance conditions (prediction verbalized on the response-relevant or the response-irrelevant stimulus dimension) as between-subject factors. The RT analysis revealed main effects of prediction match (longer RTs after prediction mismatch), $F(1,58)=227.95, p<.001, \eta^{2}=.80$, and relevance (longer RTs if a prediction had to be verbalized on the response irrelevant stimulus dimension), $F(1,58)=41.57$, $p<.001, \eta^{2}=.42$. There was a two-way interaction of prediction match and validity, $F(1,58)=15.66, p<.001, \eta^{2}=.21$, and of prediction match and relevance, $F(1,58)=55.50, p<$ $.001, \eta^{2}=.49$. There was no three-way interaction $(F<1)$. The difference between prediction match and mismatch was larger if the prediction specified the response-relevant stimulus feature, and it was larger if a prediction was more valid (i.e., if the stimulus matched the prediction on a higher proportion of the trials).

Separate analyses on the data of the four experimental groups (relevant-low, relevant-high, irrelevant-low, irrelevant-high) revealed that the prediction effect on RT was 
a

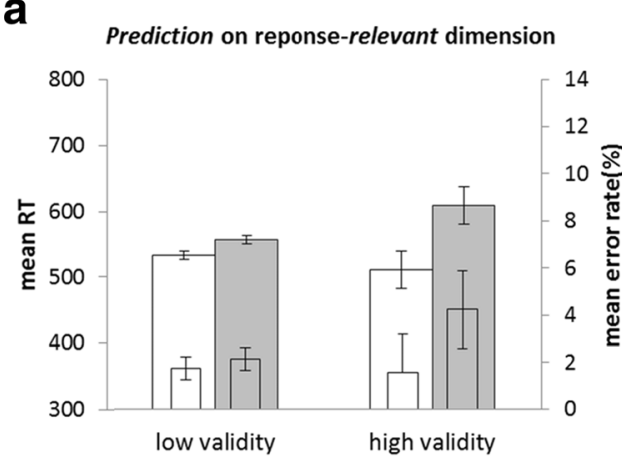

C

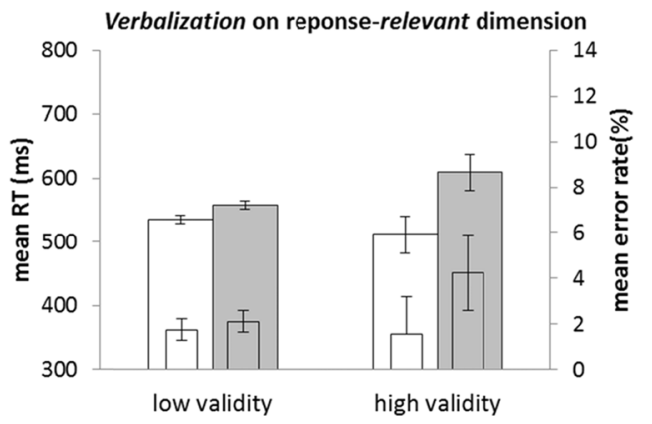

Fig. 2 Mean response times (wide bars) and error rates (narrow bars) for predictions on the response-relevant stimulus dimension (a) or response irrelevant stimulus dimension (b) of Experiment 1a. Panel $\mathbf{c}$ shows response times in conditions eliciting verbalizations on the response-

significant in all conditions (all $p \mathrm{~s}<.01$ ). Even when the prediction was (a) not response relevant and (b) was not valid, participants seemed to include the prediction they just generated in their preparation for the upcoming trial.

While the mean error rate was low $(2.25 \%, S D=1.89)$, the pattern was similar in part to the one observed in the reaction times. There was a main effect of prediction match, $F(1,58)=$ 20.67, $p<.001, \eta^{2}=.26$. Error rates showed an interaction between prediction match and relevance, $F(1,58)=16.45, p<$ $.001, \eta^{2}=.22$, and an interaction of prediction match and validity, $F(1,58)=16.40, p<.001, \eta^{2}=.22$. Different from the RTs, mean error rate was generally higher in the $80 \%$ valid group, $F(1,58)=6.25, p=.015, \eta^{2}=.097$, but did not differ with respect to response relevance of the predictions. The prediction effect in the error rates reversed when the prediction was irrelevant for response selection. Thus, while participants predicting a response-irrelevant feature thus were faster in match trials than in mismatch trials, they were more error prone in match trials than in mismatch trials. Because of this tradeoff, the RT effect should be interpreted with caution. Presumably, a match between response-irrelevant prediction and stimulus feature might have acted as a go signal for executing a prepotent response, thereby causing errors. In line with this post hoc explanation, exploratory analyses showed that the error rate for the fastest $20 \%$ reactions differed between the low $(6.88 \%)$ and the high $(3.04 \%)$ validity b

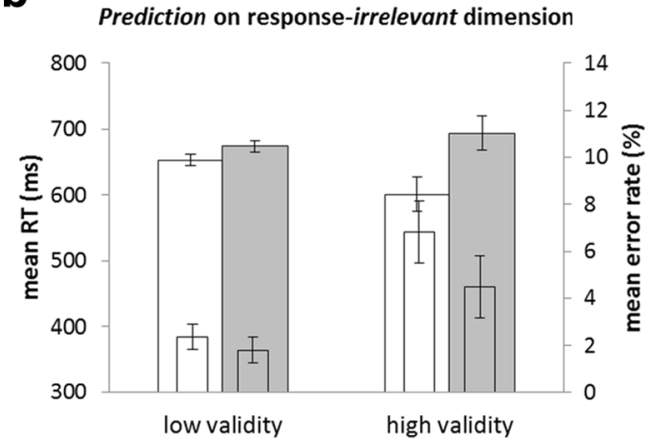

d

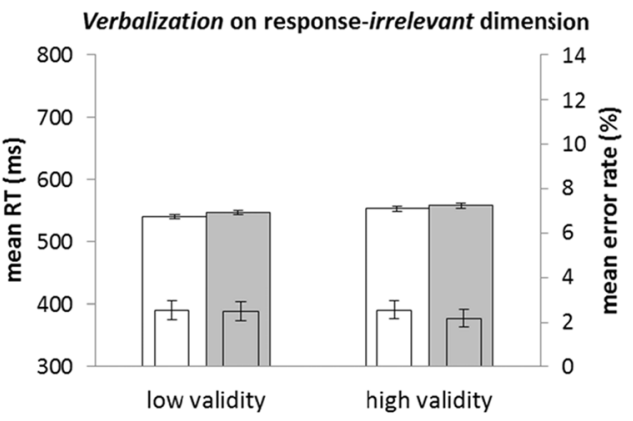

relevant and on the response-irrelevant dimension (d) of Experiment 1b. Errors bars mark the confidence intervals for the within-subjects effects of prediction match

condition, $t(29)=2.696, p=.012$; no differences in the other quintiles. In the low-validity condition, a match between the predicted response-irrelevant feature and the stimulus was a rare event, potentially triggering the execution of a prepotent response.

Quintile analyses In line with an effect on weighting of the prediction for response preparation, validity and response relevance shifted the entire RT distribution (see Appendix A).

Sequential match effects We analyzed the impact of match/ mismatch in the previous trial on the reaction time match effect in the current trial in a mixed ANOVA, including the within subjects factors prediction (match vs. mismatch in current trial) and previous prediction (match vs. mismatch in preceding trial) as well as the between-subjects factors validity and relevance (see Appendix B for mean values per condition). Violation of prediction led to a general slowing of responses in the next trial (main effect of previous prediction), $F(1,58)=15.98, p<.001, \eta^{2}=.22$. The prediction effect on reaction times was reduced if the stimulus in the preceding trial mismatched the prediction: there was an interaction between prediction and previous prediction, $F(1,58)=7.40, p=$ $.009, \eta^{2}=.11$. There were no significant interactions with the between-subjects factors, indicating no robust difference of the sequential effect amongst the four experimental groups 
( $p$ s > .25; the match effect was reduced by $24 \mathrm{~ms}$ in the relevant-low group, by $30 \mathrm{~ms}$ in the relevant-high group, by $7 \mathrm{~ms}$ in the irrelevant-low group, and by $15 \mathrm{~ms}$ in the irrelevanthigh group).

In the error analysis, the two-way interaction of prediction and previous prediction was not robust, $F(1,58)=3.31, p=$ $.074, \eta^{2}=.05$. However, there was a significant three-way interaction involving the between-subjects factor relevance, and the within-subject factors prediction and previous prediction: $F(1,58)=7.14, p=.010, \eta^{2}=.11$. There was also a three-way interaction involving the between-subjects factor validity as well as prediction and previous prediction, $F(1,58)$ $=4.42, p=.040, \eta^{2}=.07$. The four-way interaction of relevance, validity, prediction, and previous prediction, $F(1,58)=$ $4.10, p=.047, \eta^{2}=.07$, mirrors the fact that previous prediction affected errors only in the relevant-high group. In this group, error rate was markedly increased if a prediction mismatch followed a match trial.

When trials containing feature repetitions on the predicted dimension were excluded from the analysis, the pattern of effects stayed the same for the response times. With error rates, there was no longer an influence of previous prediction.

Validity estimation Because of an error in the program, we failed to inquire the first 20 participants about their validity estimate at the end of the experiment. Though we used behavioral data of 62 participants, validity estimates were obtained from 42 participants (11 and 10 in the low-validity relevant and irrelevant condition and 10 and 11 in the high-validity relevant and irrelevant condition, respectively). Estimated proportion of trials in which the stimulus matched the prediction was significantly higher in the high-validity groups (on average $75 \%$ in the relevant-high group and $71 \%$ in the irrelevant-high group) than in the low-validity conditions (relevant-low: 30\%, irrelevant-low: 27\%; main effect of validity), $F(1,42)=91.35, p<.001, \eta^{2}=.71$. The proportion of matches, was underestimated significantly in the $80 \%$ match condition, $t(20)=-2.61, p=.017$. In the low-validity condition the underestimation was not significant, $t(20)=-1.29, p=$ .211 .

\section{Discussion}

In Experiment 1a, we found stronger prediction effects for valid and relevant predictions. Thus, though chance level predictions have been documented to lead to RT effects that are comparable to RT effects for highly valid cues (see Gaschler et al., 2014, for a summary), the effect can be pushed even further by increasing the validity of predictions. With highly valid predictions, the predicted stimulus feature might be represented in the focus of attention in working memory more strongly or in a higher proportion of trials. Though prediction effects can be boosted, flexibility of the prediction-preparation link seems to be limited as prediction effects apparently can't be reduced to zero. We found a strong prediction effect for chance-valid predictions on the response-relevant stimulus dimension and for valid predictions on the response-irrelevant dimension. For predictions on the response-irrelevant dimension, RT and error rate unexpectedly showed opposing tendencies. Presumably, matches on the response-irrelevant dimension could in some trials trigger the execution of a prepotent response tendency.

In line with Mattler et al. (2006), even predictions concerning a response-irrelevant stimulus feature led to an effect. The postexperimental validity estimates showed that participants knew about the validity. Apparently, prediction had an impact on preparatory processes even when it was known to be neither useful nor relevant. In addition, aftereffects of prediction mismatch did not differ robustly between conditions. A mismatch in the low-validity condition led to a similar reduction of the prediction effect in the subsequent trial as did a (less likely) mismatch in the high-validity condition. This suggests that predictions have automatic effects and aftereffects.

\section{Experiment 1b}

To disentangle effects tied to predicting stimuli versus uttering words, we had participants of Experiment $1 \mathrm{~b}$ utter the exact same words that had been verbalized as predictions by the participants of Experiment 1a. Uttering words was introduced as a secondary task. No relation between these words and the stimuli of the manual choice task was mentioned. To the extent that the effects observed in Experiment 1a are specific to predictions per se (rather than to feature priming at work irrespective of whether verbalized features are intended to denote predictions or not), effects should be reduced in Experiment $1 b$. We hypothesized that overlap between verbalized and presented stimulus feature would lead to smaller effects than predictions in Experiment 1a for the conditions where the verbalized feature is response relevant and a lack of an effect when it is response-irrelevant. As participants automatically acquire and use correlations between stimulus features (and responses; cf. Mordkoff \& Halterman, 2008), verbalizing response-relevant features that often match the stimulus and correlate with the required response should have an effect on performance. Therefore, we expected to obtain a validity effect.

\section{Method}

Participants Sixty-six undergraduate students of psychology and other fields (42 women, mean age $=24.95$ years, $S D=$ 4.13) participated in individual sessions lasting around 
60 minutes. Participants either received partial course credit or were paid $€ 8$ for their time.

Stimuli and design The same colored shapes as in Experiment 1a served as stimuli. Words presented for reading corresponded to the predictions verbalized by the participants of Experiment 1a. Thus, the two groups (Experiments 1a and $1 \mathrm{~b})$ were yoked with respect to the words they pronounced as well as the stimuli they responded to.

Similar to Experiment 1a, the words were followed by a stimulus matching the word in the pronounced feature value on $80 \%$ of the trials or by chance (i.e., on $33 \%$ of the trials). As the second between-subjects factor, the words specified feature values either on the response-relevant or the responseirrelevant dimension.

Procedure The number and procedure of blocks and trials was identical to Experiment 1a except for the following changes: Instead of the question at the beginning of each trial a black horizontal line appeared in the center of the screen for $400 \mathrm{~ms}$ and was replaced by the word rot, grün or blau, or Stern, Haus or Kreuz, respectively. After the participant had read the word aloud, the experimenter keyed it in. After $2,100 \mathrm{~ms}$, the word was replaced by the imperative stimulus, and the participant chose the appropriate response key as fast as possible. The next trial began $500 \mathrm{~ms}$ after the response or after reaching the response deadline of 2,500 ms.

After the final experimental block the experimenter asked participants to estimate the proportion of experimental trials overall in which the stimulus matched the word they had read at the beginning of the trial.

\section{Results}

The data of two participants were excluded from further analyses because their error rate exceeded $10 \%$. From the data of the remaining participants, trials with no registered verbalization were excluded (on average $0.4 \%, S D=0.80$ ). Validity estimation results are based on 62 participants (two participants stated that they were unable to give an estimation answer).

Mean response time in Experiment $1 \mathrm{~b}$ was $546 \mathrm{~ms}(S D=$ $71.23)$, mean error rate was $2.3 \%(S D=1.36)$. Thus, general performance was similar to Experiment 1a, which is not surprising because the choice reaction task was identical. The critical question concerns the match effects (see Figs. $2 \mathrm{c}$ and d). The ANOVA with word match as within-subject factor and validity and relevance as between-subjects factors revealed a main effect of word match on response times, $F(1,60)=$ $43.16, p<.001, \eta^{2}=.42$, as well as on error rates, $F(1,60)$ $=4.45, p=.039, \eta^{2}=.07$. The size of the effect depended on predictive validity of the word participants read aloud before the choice stimulus, RTs: $F(1,60)=13.84, p<.001, \eta^{2}=.19$;
ERs: $F(1,60)=2.53, p=.12, n s, \eta^{2}=.04$, as well as relevance, RTs: $F(1,60)=29.43, p<.001, \eta^{2}=.33$; ERs: $F(1,60)=7.51, p=.008, \eta^{2}=.11$. Beyond the two-way interactions, the three-way interaction of word match, validity, and relevance was also significant for response times, $F(1,60)$ $=14.22, p<.001, \eta^{2}=.19$, and errors rates, $F(1,60)=4.42, p$ $=.04, \eta^{2}=.07$. As in Experiment 1a, performance was better when the stimulus matched the feature specified by the verbalized word. This difference was larger when the feature was relevant for choosing the response and when the probability of match was high. The three-way interaction resulted from the fact that the match effect diminished to 6 milliseconds (both in the high and in the low validity condition) if the word related to the response-irrelevant stimulus dimension (see Fig. 3 for the comparison of the mismatch-match difference in Experiment 1a vs. 1b). Though freely verbalizing response irrelevant stimulus features as predictions (Experiment 1a) led to a match effect, reading aloud words corresponding to response irrelevant stimulus features did not (Experiment 1b).

Sequential match effects Similar to Experiment 1a, there was a significant reduction in the RT match effect following mismatch trials (Word Match $\times$ Previous Match): $F(1,60)=$ $11.63, p=.001, \eta^{2}=.16$. It was only present when the word to be read related to the response-relevant stimulus dimension (Word Match $\times$ Previous Match $\times$ Relevance): $F(1,60)=$ $13.18, p=.001, \eta^{2}=.18$. The reduction in the match effect amounted to $34 \mathrm{~ms}$ in the relevant-low group, $30 \mathrm{~ms}$ in the relevant-high group, $4 \mathrm{~ms}$ in the irrelevant-low group, and $-6 \mathrm{~ms}$ in the irrelevant-high group. With mean error rates, the match effect was modulated by a mismatch in the preceding trial (interaction Word Match $\times$ Previous Match): $F(1,60)$ $=5.63, p=.021, \eta^{2}=.09$; other $F_{\mathrm{S}}<1$. Note that when feature repetitions on the response-relevant dimension are excluded, the adaptation effect (interaction Word Match $\times$ Previous

relevant word

irrelevant word

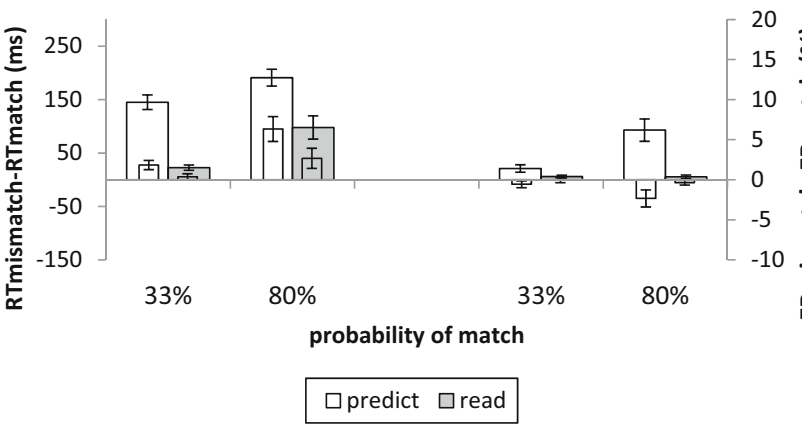

Fig. 3 Mean prediction effect as response time-difference (wide bars) and error rates-difference (narrow bars) for participants who predicted stimulus features (Exp. 1a) or read aloud words that specified certain stimulus features (Exp.1b) on the response-relevant versus the responseirrelevant dimension. Errors bars represent the standard error of the group mean 
Match) remains significant in response times whereas it is no longer significant in error rates $(p>.1)$.

Proportion match estimation The proportion of trials in which the stimulus matched the word was estimated significantly higher in the $80 \%$ match conditions, $F(1,62)=27.13, p$ $<.001, \eta^{2}=.32$, and there was an interaction of validity and relevance, $F(1,62)=4.23, p=.044, \eta^{2}=.07$. The estimates were on average $30 \%$ (relevant-low), $38 \%$ (irrelevant-low), 63\% (relevant-high), and 53\% (irrelevant, high). Estimation did not differ significantly from the true match probabilities in the low validity groups, relevant: $t(16)=-1.00, p=.331$; irrelevant: $t(14)=1.14, p=.274$. In the conditions with $80 \%$ matches the probability was consistently underestimated, relevant: $t(13)=-3.23, p=.007$; irrelevant: $t(15)=-5.16, p<$ .001 .

\section{Discussion}

Predictions in Experiment 1a had an impact on performance even under adverse circumstances (low validity and response irrelevant prediction). Experiment $1 \mathrm{~b}$ suggests that these effects were in part specific to predictions. Mismatch effects were only present when the words specified a responserelevant stimulus feature and were smaller than for uttering the same words as a prediction. Larger effects in the condition with $80 \%$ match between verbalized word and (response relevant) stimulus feature might be caused by participants noticing the relation if the stimulus feature is response relevant. This might have changed the secondary read-aloud task to a verbal cueing task.

As in Experiment 1a, the sequential match effect showed characteristics of an automatic aftereffect. It was present to a similar extent in both conditions with response-relevant verbalizations.

\section{Experiment 2}

In Experiment 1a we observed that predictions had an impact on task processing even under adverse conditions (chance validity or response irrelevance). This suggests that the impact of predictions on task processing is in part automatic. However, preparation effects were also larger for valid and response-relevant predictions. This might suggest that the link between prediction and preparation is in part under strategic control. Because we manipulated response relevance and validity between groups, we cannot assess whether participants can flexibly vary on a trial-by-trial basis whether their predictions influence task processing.

In Experiment 2 we manipulated predictions more directly, rather than their relevance or validity. We used a frequency manipulation to influence predictions. Three feature alternatives occurred with different frequencies (on $60 \%$, $30 \%$, or $10 \%$ of the trials). This should lead participants to predict the most frequent feature more frequently than the other two and allow us to assess strategic flexibility in choosing and implementing predictions. First, participants might use the task to verbalize predictions strategically in order to maximize performance. Given the large RT match effects, they could maximize performance by predicting the most frequent stimulus on every trial. Second, if participants refrain from consistently predicting the most frequent stimulus, they might still show strategic flexibility concerning the predictionpreparation link. When predicting the most frequent stimulus (which is a prediction likely met by the stimulus), they should engage in task preparation, while they should not when predicting the infrequent stimulus. In principle, with full flexibility in the prediction-preparation link, they could even prepare for the frequent stimulus when predicting the infrequent one, as the former is most likely to occur (cf. Jiménez \& Méndez, 2013; Umbach, Schwager, Frensch, \& Gaschler, 2012, for dissociation between prediction and preparation).

\section{Method}

Participants A total of 28 undergraduate students of psychology and other fields ( 13 women, mean age $=25.23$ years, $S D$ $=4.95$ ) participated in individual sessions lasting around 60 minutes. Participants either received partial course credit or $€ 8$ compensation.

Stimuli, design, and procedure Participants predicted the response-relevant stimulus feature. Stimuli were identical to Experiment 1 . Unlike in the high-validity condition of Experiment 1, stimulus selection was not contingent upon prediction. Instead, we manipulated the frequency of the response-relevant feature-frequent $(60 \%)$, less frequent $(30 \%)$, and infrequent $(10 \%)$. Assignment of features to frequency conditions was balanced across participants.

Participants completed five blocks of 120 trials for a total of 600 trials (72 frequent, 32 less frequent, and 12 infrequent trials per block). Rather than having an experimenter handcode verbalizations online while sitting next to the participant, this time verbal predictions were captured with a microphone headset and identified using a real-time speech recognition program implemented in MATLAB (Donkin, Brown, \& Heathcote, 2009). At the beginning of the experimental session, the software was trained to the individual voice with the participant speaking aloud the words in the response set (Stern, Haus, Kreuz, or rot, blau, grün) 10 times. This was followed immediately by an accuracy check with 10 additional exemplars per word. If recognition accuracy was below 95\% (i.e., more than one misidentification) the original training was restarted, otherwise the additional exemplars were added to the pool of training exemplars and the experiment 
commenced. Recognition accuracy was tested again in the middle and at the end of the session. Participants were reminded in random intervals (on about every 10th trial) to speak loudly and clearly.

\section{Results}

Data of six participants were excluded from further analysis because the accuracy of speech recognition failed the $75 \%$ criterion in the check at the end of the session. No further datasets had to be excluded (response accuracy in the choice task was above $90 \%$ in all participants). For the remaining data, mean speech recognition accuracy was $99 \%, 95 \%$, and $91 \%$ (at the start, in the middle, and at the end of the session, respectively).

Frequency effects in predictions and responses In a first step, we considered the frequencies with which participants predicted feature values of the three different frequency classes. Figure 4a shows that already in Block 1, the most frequent value is predicted most often, followed by the less frequent and the infrequent value. An ANOVA revealed a main effect of the factor frequency, $F(2,42)=17.54, p<.001, \eta^{2}=.46$. This difference enlarged over blocks (interaction Frequency $\times$ Block): $F(8,168)=4.56, p<.001, \eta^{2}=.18$, as with practice, participants came to match the probabilities of occurrence in their predictions. Bonferroni corrected pairwise comparisons showed that the difference between the prediction frequency of the $60 \%$ and $30 \%$ feature was statistically significant from Block 2 onwards. The difference between prediction frequency of the $30 \%$ and the $10 \%$ feature was not significant (minimum $p=.08$ in Block 4). Frequency effects on speed and accuracy of responses developed similarly: Besides a practice effect (main effect of block; $R T s$ ): $F(4,84)=6.06, p<.001, \eta^{2}$ $=.22$; ERs: $F<1$, there was a main effect of frequency, RTs: $F(2,42)=48.51, p<.001, \eta^{2}=70$; ERs: $F(2,42)=7.81, p=$ $.001, \eta^{2}=.27$, with more frequent stimuli leading to faster and more accurate reactions. The frequency effect increased with practice, leading to a significant interaction of frequency and block, RTs: $F(4,84)=4.82, p<.001, \eta^{2}=.19$; ERs: $F<1$; see Figure $4 \mathrm{~b}$.

Prediction effects The design of Experiment 2 is not suitable for running analyses that include all three factors (block, frequency and prediction match) because there were not enough infrequent stimuli per prediction match condition and block to allow for reliable estimates within a participant. We therefore used aggregated data in two two-way ANOVAs.

Figure 5 shows means of median response times and mean error rates per condition and person. An ANOVA on the RTs with frequency and prediction match as within-subject factors revealed main effects of both factors, frequency: $F(2,42)=$ $30.03, p<.001, \eta^{2}=.59 ;$ prediction match: $F(1,21)=197.70$,
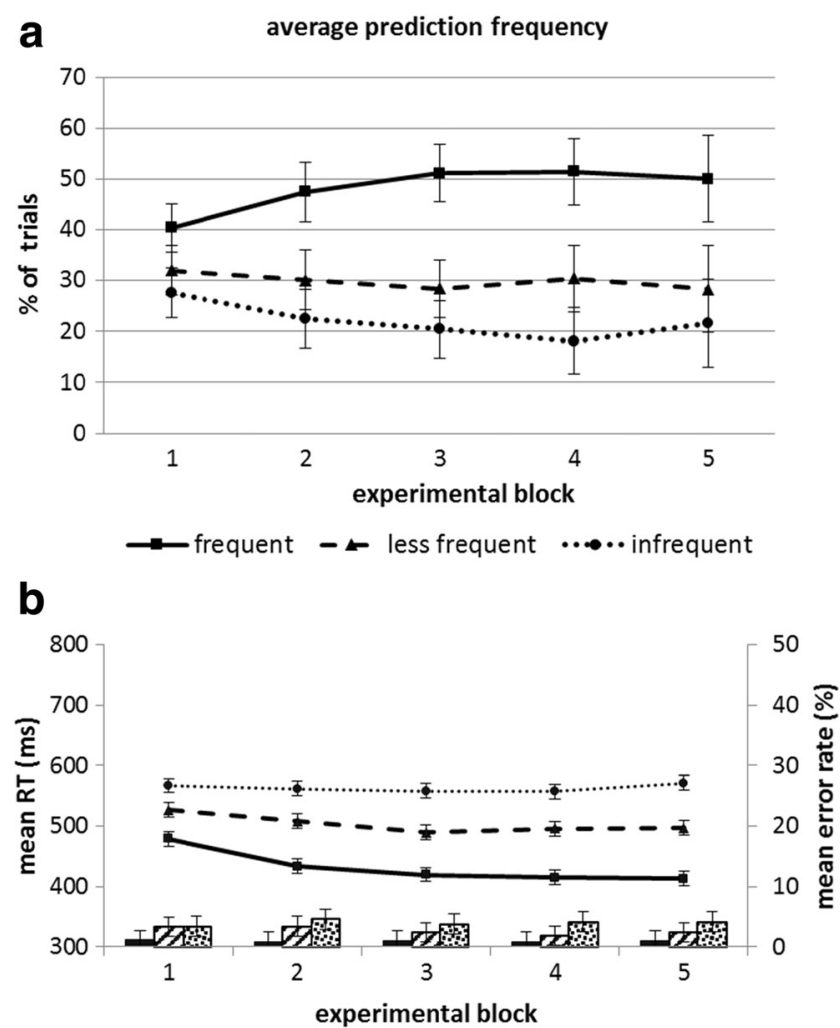

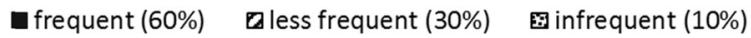

Fig. 4 Mean proportions of stimulus feature predictions for feature values that occurred on $60 \%, 30 \%$, or $10 \%$ of trials (a). Panel b shows the mean RTs and ERs for frequent (solid line / black bars), less frequent (dashed line / striped bars), and infrequent (dotted line / dotted bars) stimulus features, over the five experimental blocks. Errors bars mark the confidence intervals for the within-subjects effects of block and frequency

$p<.001, \eta^{2}=.90$, but no interaction $(F<1)$. Responses were faster for stimuli presented more frequently, and they were faster when the prediction was met by the stimulus. Remarkably, the prediction effect appears to be independent of stimulus frequency.

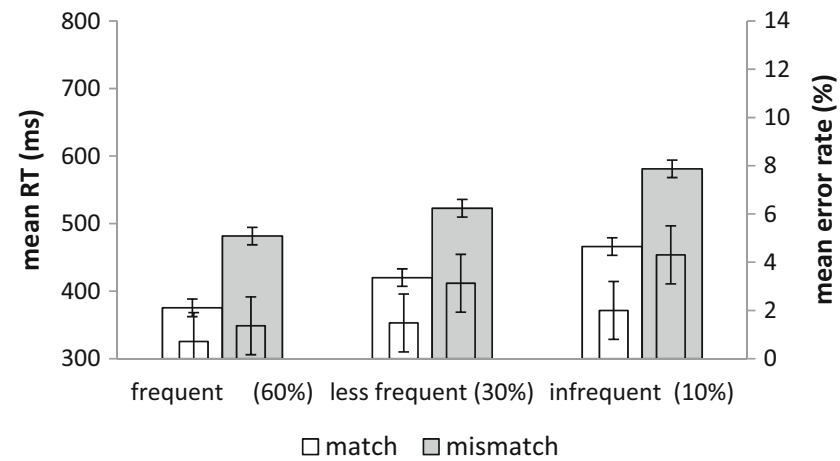

Fig. 5 Mean response times (wide bars) and errors rates (narrow bars) for trials in which the stimulus matched/mismatched the prediction verbalized by the participant, separately for the three frequency classes of stimulus features. Error bars mark the confidence interval of the withinsubject interaction of frequency and prediction match 
As mean error rates were quite low in this experiment, they are less informative than RTs, but all in all they showed the same effects, main effect of frequency: $F(2,42)=6.97, p=$ $.002, \eta^{2}=.25$; main effect prediction match, $F(1,21)=9.33, p$ $=.006, \eta^{2}=31$; no interaction: $F<1$. Thus, there was no hint of a speed-accuracy trade-off that could have blurred the RT effects.

Because probability matching improves over the first three blocks, it is conceivable that participants relied more on their predictions in later blocks. In Fig. 6, performance data are plotted for the five experimental blocks, averaged across frequency conditions. ANOVAs revealed a stable prediction match effect, RTs: $F(1,21)=306.80, p<.001, \eta^{2}=.94$; ERs: $F(1,21)=12.70, p=.002, \eta^{2}=.38$, and improvements in response speed with practice, main effect block on RTs: $F(4$, $84)=7.88, p<.001, \eta^{2}=.27$; on ERs: $F<1$, but no interaction $(F \mathrm{~s}<1.1)$.

Sequential match effects We found a prediction adaptation effect (the prediction effect was modulated by match or mismatch of the preceding trial in RTs (interaction of prediction match and previous prediction match), RTs: $F(2,42)=32.93, p<.001, \eta^{2}=.61$, as well as in ERs, $F(2,42)=5.63, p=.021, \eta^{2}=.21$. The reduction in the response time effect was on average $32 \mathrm{~ms}$, caused by slower RTs in match trials following a mismatch. In an additional exploratory analysis we looked for differences in adaptation after predictions of more or less frequent stimuli. Trials in which rare stimuli were predicted were excluded from this analysis because there were too few (often no) trials with two successive matches; two more participants had fewer than five of such cases in the less-frequent condition, too, and were also excluded. The adaptation effect in this reduced sample did not differ significantly with respect to whether frequent or less frequent stimuli were

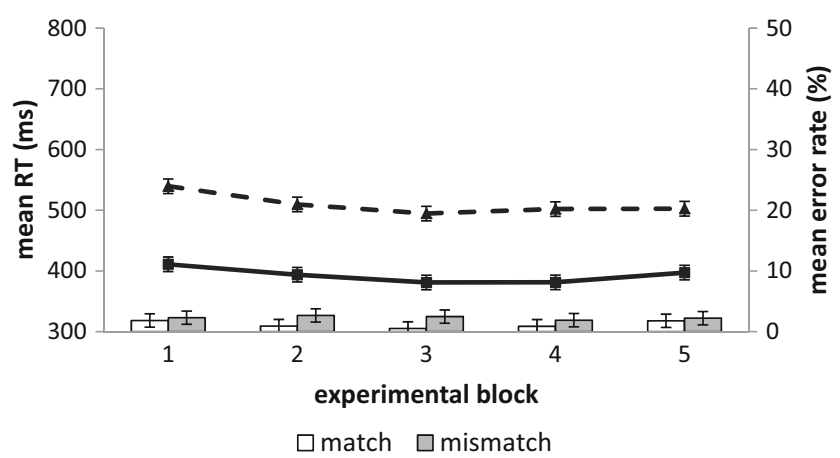

Fig. 6 Mean RTs and ERs in Blocks 1 to 5 for trials in which the stimulus matches (solid line) or mismatches (dashed line) the participant's prediction, averaged across frequency conditions. Error bars mark the confidence interval of the within-subject interaction of block and prediction match predicted (Frequency $\times$ Prediction Match $\times$ Previous Match: $F<1)$.

\section{Discussion}

Experiment 2 casts into doubt that participants flexibly use predictions to boost performance. First, their predictions showed probability matching rather than maximizing. Given the large RT benefit linked to correct predictions, participants could have boosted performance by strategically opting for predicting the most frequent stimulus on all trials. Gray, Sims, Fu, and Schoelles (2006) suggested that RT effects of the size observed here are large enough to lead to strategy adjustments. Second, participants did not seem to vary on a trial-by-trial basis to what extent they used predictions for preparation. When predicting the infrequent or less frequent stimulus they seemed to prepare for exactly this stimulus, while performance would have been optimized by preparing for the most frequent stimulus instead. Note that dissociations between prediction and preparation are possible (e.g., Jiménez \& Méndez, 2013). Umbach et al. (2012) showed that participants can keep up with a response deadline assigned to a less frequent stimulus while predicting the frequent stimulus to occur. Third, aftereffects of prediction (mis)match did not seem to vary with frequency: When predicting the most frequent stimulus prediction, mismatch seemed to have the same consequences for the next trial as when predicting the less frequent stimulus, despite much more likely mismatches in the latter case. Finally, prediction effects were stable with practice.

\section{General discussion}

In this study we tested how flexibly people could control the link between predicting a stimulus and preparing for it. While it was known that strong RT and EEG differences result, when the stimulus by chance matches rather than mismatches selfgenerated verbalized prediction of a trial (e.g., Acosta, 1982; Kemper et al. 2012), a systematic test of how flexibly participants could (a) choose predictions to optimize performance and (b) control whether to implement predictions was lacking so far. Moving beyond demonstrations of prediction effects occurring when stimuli are drawn randomly (e.g., Gaschler et al., 2014, for an overview), Experiment 1 provides the first systematic variation of validity and response relevance of stimulus predictions. On the one hand, results showed that the prediction effect can be boosted further when predictions are valid (i.e., $80 \%$ chance of match between prediction and stimulus). On the other hand, the prediction effect did not fully disappear under adverse circumstances (i.e., when predicting a response-irrelevant feature). Analyses of the RT distribution suggested that validity and response relevance of the 
predictions seemed to increase the weight of predictions for response preparation. In line with the intentional weighting principle (e.g., Memelink \& Hommel, 2013), we observed a general shift of the RT distribution. The impact of validity and response relevance of predictions on performance was particularly pronounced in match trials. This is consistent with the interpretation that predicting the stimulus results in a benefit, if the stimulus is actually presented, while costs occur in mismatch trials as the prediction-based advance activation of the stimulus representation has to be overcome.

Experiment 2 provided further evidence for the notion that participants can weight the extent to which their predictions impact preparation only at a general level rather than trial by trial (cf. Pfister et al., 2010). Despite receiving extended practice with a set of stimuli varying in frequency, participants did not start to strategically choose predictions to optimize performance. Rather than focusing on the most frequent stimulus for predictions (which would have optimized the proportion of match trials and efficiency of task processing), participants showed sustained probability matching (cf. Gaissmaier \& Schooler, 2008). Apart from choosing predictions, participants did not flexibly use the prediction-preparation link either in terms of implementing predictions. Despite being aware of stimulus frequencies, they showed a prediction effect of similar size for infrequent, medium, and frequent stimuli. Particularly, the infrequent stimulus was processed faster in the rare case that it was (a) predicted and (b) presented. The most likely event (and therefore target of preparation optimizing task performance) would have been the frequent stimulus instead.

Strong effects of predictions despite adverse circumstances (chance-level or response-irrelevant stimulus feature) support the view that stimulus feature representations gain a privileged status when being verbalized as predictions. The results are consistent with the view that the content of a prediction is represented in the focus of attention in working memory (cf. Gaschler et al., 2014). The current systematic variation of validity and response relevance of predictions could provide some insight into the implications of this account and allows us to explore potential mechanisms that mediate the impact of predictions on preparation. Two mechanisms can be conceived, by which validity and relevance modulate the prediction-preparation link. The percentage of trials in which the predicted stimulus representation actually gains the privileged status could be a function of validity or relevance (cf. Lindsen \& de Jong, 2010). Alternatively, the representation of predicted stimuli in general could be weighted differently depending on relevance or validity (cf. Memelink \& Hommel, 2013). RT distributions in Experiment 1 are consistent with the latter view.

We attribute prediction-based response time advantages mainly to advantages in stimulus processing due to advance activation of the stimulus representation. Kemper et al. (2012) used a 2:1 mapping of stimuli to responses. Responses were fast if the predicted stimulus was presented (so that the response mapped to this stimulus could be executed). Participants were slowed to a similar extent in either of the two possible variants of mismatch: (1) presentation of a stimulus that was (a) not predicted and (b) mapped to a different response than the predicted stimulus and (2) presentation of a stimulus not predicted, but mapped to the same response as the predicted stimulus. The lateralized readiness potential (LRP) of the EEG suggested that response activation was tied to the imperative stimulus. In case of a stimulus that did not match the prediction but called for the same response as the predicted stimulus, initial response preparation was cancelled and reset when the stimulus appeared, even though the response activation was the one needed to complete the trial. Similarly, EEG data in Mattler et al. (2006) indicated that response preparation engaged in prior to stimulus onset could be canceled in case of a mismatch between stimulus and stimulus cue. An LRP of the expected response was observed prior to stimulus presentation and increased further if stimulus information was as expected. Otherwise, preparation was cancelled. The adjusted expectancy model (Mattler, 2005) thus provides an account for the regulation of expectation effects within trials (i.e., dominance of attended stimulus dimension over potential response match) and might also provide a basis for explaining prediction aftereffects. Potentially, reliance on predictions is reduced for more than just the current trial in case of a mismatch.

Note, however, that future studies need to elaborate whether sequential match effects are indeed related to predictions. They also occurred in Experiment $1 \mathrm{~b}$ where participants had to read aloud as a secondary task in a yoked design what participants in Experiment 1a had verbalized as self-generated predictions. Aftereffects of prediction match (Experiments 1a and 2) and aftereffects of a match between a word denoting a response-relevant feature and the stimulus (Experiment 1b) were of similar size irrespective of stimulus frequency and frequency of match between verbalization and stimulus. The sequential match effect was not affected by how likely a mismatch was. Rather, it was present whenever a response-relevant feature had been verbalized. Potentially, overlap between verbalization and stimulus increased the weight of the response-relevant stimulus feature relative to the response-irrelevant feature, while mismatch might have had the opposite effect. Consequently, one might suggest that the sequential match effect is not an aftereffect of prediction per se, but rather an automatic aftereffect tied to feature overlap between verbalization and response. A feature priming explanation, however, might not be sufficient for all cases of sequential modulation of prediction effects. Duthoo, Wühr, and Notebaert (2013; see also Kemper, 
Gaschler, Schwager, \& Schubert, 2016) observed a sequential modulation with conflict level predictions. In this case the prediction does not denote a specific response feature, so feature overlap can't cause the effect. Furthermore, it is interesting to note that the sequential modulation of the match effect showed predominantly in match trials and was smaller in mismatch trials, suggesting adaptation to the lack of conflict (rather than to conflict) in Kemper et al. (2016). Similarly, in this study, match versus mismatch between verbalization and stimulus in the preceding trial had most of an influence if the current trial was a match trial (see Appendix B).

Taken together, the experiments suggest that people exert only limited strategic control over the link between prediction and task preparation. Internally generated and verbalized expectations (i.e., predictions) might be coupled to preparatory processes especially tightly.

Acknowledgements We thank Björn Elsner and all other members of our lab for their help in conducting the experiments as well as providing helpful feedback throughout the preparation of the manuscript. This research was supported by the German Research Foundation (DFG) Grant FR 1471/9-2 awarded to Peter A. Frensch.

\section{Appendix A: Quintile analyses}

To explore whether validity and relevance affected the weighting of the prediction for response preparation, or rather the proportion of trials in which the prediction was fully used for response preparation, we analyzed the reaction time distribution of match trials. We reasoned that while mismatch trials should reflect effects of advance activation of a stimulus representation as well as conflict with the stimulus actually presented, match trials seem to represent prediction based preparation more directly.

A general impact of validity on the amount of prediction-based preparatory processes should result in a general shift of the RT distributions of high versus low validity (i.e., parallel lines in the quintile analysis, as shown in Fig. 7). If, however, the validity effect was a mixture effect (larger proportion of trials in which the representation gained access to the focus of attention in working memory and thereby full impact on preparatory processes), one could assume that at least the fastest quintiles of match trials in the high- and low-validity conditions should overlap. A similar approach to differentiate between a mixture-based and a weight-based explanation has been taken by Lindsen and de Jong (2010) to differentiate between residual switch costs in task switching stemming from (a) partial preparation in essentially all trials versus (b) a mixture of some trials fully prepared in advance and other trials not prepared at all.

The parallel lines in Fig. 7 seem consistent with the intentional weighting principle (e.g., Memelink \& Hommel, 2013). Match trials of participants predicting the response-relevant feature were significantly faster than match trials of participants predicting the response-irrelevant feature $(p s<.001)$. More importantly, when the prediction concerned the response-relevant stimulus feature, there was a significant advantage of the high-validity condition match trials over lowvalidity condition match trials in all quintiles $(p<.05$ two-tailed in Quintiles 1, 2, 3 and 5, $p<.05$, one-tailed for Quintile 4). For match trials in predicting the response irrelevant stimulus feature, quintile differences for validity were not significant $(p s>.24)$.

Note also that the difference between match and mismatch trials was significant for each condition and in each quintile $(p s<.022)$. Even in the low validity response irrelevant group, which had shown overall the weakest prediction effect in the RTs, the prediction match effect was significant in all quintiles. This suggests that prediction and mismatch processing affect the entire RT distribution rather than, for instance, only the fastest or slowest responses (e.g., in case of strong preparation in just a minority of trials, a strong benefit in case of a

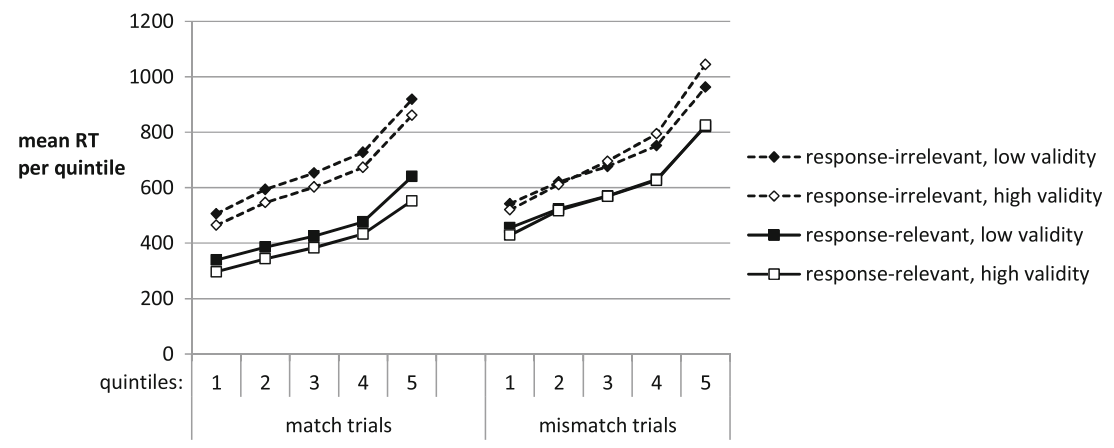

Fig. 7 Mean reaction time per reaction time quintile per condition 
Table 1 Sequential match effects

\begin{tabular}{|c|c|c|c|c|c|c|c|c|c|c|c|c|c|}
\hline \multirow{3}{*}{$\begin{array}{l}\text { (a) } \\
\text { Current trial: }\end{array}$} & \multicolumn{13}{|c|}{ Experiment 1a (predict) } \\
\hline & & \multicolumn{3}{|c|}{$\begin{array}{l}\text { Relevant dimension low } \\
\text { validity }\end{array}$} & \multicolumn{3}{|c|}{$\begin{array}{l}\text { Relevant dimension high } \\
\text { validity }\end{array}$} & \multicolumn{3}{|c|}{$\begin{array}{l}\text { Irrelevant dimension low } \\
\text { validity }\end{array}$} & \multicolumn{3}{|c|}{$\begin{array}{l}\text { Irrelevant dimension high } \\
\text { validity }\end{array}$} \\
\hline & & Match & Mismatch & $\begin{array}{l}\text { Match } \\
\text { effect }\end{array}$ & Match & Mismatch & $\begin{array}{l}\text { Match } \\
\text { effect }\end{array}$ & Match & Mismatch & $\begin{array}{l}\text { Match } \\
\text { effect }\end{array}$ & Match & Mismatch & $\begin{array}{l}\text { Match } \\
\text { effect }\end{array}$ \\
\hline Preceding & Match & 401 & 562 & 161 & 376 & 574 & 198 & 647 & 670 & 23 & 597 & 695 & 98 \\
\hline trial: & Mismatch & 433 & 571 & 138 & 407 & 575 & 168 & 660 & 676 & 16 & 616 & 699 & 83 \\
\hline \multirow{2}{*}{\multicolumn{2}{|c|}{$\begin{array}{l}\text { Adaption effect } \\
\text { Experiment } 1 \mathrm{~b} \text { (read) }\end{array}$}} & \multicolumn{3}{|l|}{23} & \multicolumn{3}{|l|}{30} & \multicolumn{3}{|l|}{7} & \multicolumn{3}{|l|}{5} \\
\hline & & \multicolumn{3}{|c|}{$\begin{array}{l}\text { Relevant dimension low } \\
\text { validity }\end{array}$} & \multicolumn{3}{|c|}{$\begin{array}{l}\text { Relevant dimension high } \\
\text { validity }\end{array}$} & \multicolumn{3}{|c|}{$\begin{array}{l}\text { Irrelevant dimension low } \\
\text { validity }\end{array}$} & \multicolumn{3}{|c|}{$\begin{array}{l}\text { Irrelevant dimension high } \\
\text { validity }\end{array}$} \\
\hline Current trial: & & Match & Mismatch & $\begin{array}{l}\text { Match } \\
\text { effect }\end{array}$ & Match & Mismatch & $\begin{array}{l}\text { Match } \\
\text { effect }\end{array}$ & Match & Mismatch & $\begin{array}{l}\text { Match } \\
\text { effect }\end{array}$ & Match & Mismatch & $\begin{array}{l}\text { Match } \\
\text { effect }\end{array}$ \\
\hline \multirow{2}{*}{$\begin{array}{l}\text { Preceding } \\
\text { trial: }\end{array}$} & Match & 508 & 556 & 48 & 505 & 614 & 109 & 545 & 554 & 9 & 554 & 558 & 4 \\
\hline & Mismatch & 543 & 557 & 14 & 528 & 606 & 78 & 540 & 546 & 6 & 553 & 564 & 11 \\
\hline \multicolumn{2}{|l|}{ Adaption effect } & \multicolumn{3}{|l|}{34} & \multicolumn{3}{|l|}{31} & \multicolumn{3}{|l|}{3} & \multicolumn{3}{|l|}{-7} \\
\hline Current trial: & & Match & Mismatch & $\begin{array}{l}\text { Match } \\
\text { effect }\end{array}$ & & & & & & & & & \\
\hline \multirow{5}{*}{$\begin{array}{l}\text { Preceding } \\
\quad \text { trial: } \\
\text { Adaption effect } \\
\text { (b) }\end{array}$} & Match & 370 & 511 & 141 & & & & & & & & & \\
\hline & Mismatch & 401 & 509 & 108 & & & & & & & & & \\
\hline & & \multicolumn{12}{|c|}{33} \\
\hline & Experimen & t la (pre & dictions) & & & & & & & & & & \\
\hline & & \multicolumn{3}{|c|}{$\begin{array}{l}\text { Relevant dimension low } \\
\text { validity }\end{array}$} & \multicolumn{3}{|c|}{$\begin{array}{l}\text { Relevant dimension high } \\
\text { validity }\end{array}$} & \multicolumn{3}{|c|}{$\begin{array}{l}\text { Irrelevant dimension low } \\
\text { validity }\end{array}$} & $\begin{array}{l}\text { Irreleva } \\
\text { valid }\end{array}$ & $\begin{array}{l}\text { ant dimensio } \\
\text { dity }\end{array}$ & n high \\
\hline Current trial: & & Match & Mismatch & $\begin{array}{l}\text { Match } \\
\text { effect }\end{array}$ & Match & Mismatch & $\begin{array}{l}\text { Match } \\
\text { effect }\end{array}$ & Match & Mismatch & $\begin{array}{l}\text { Match } \\
\text { effect }\end{array}$ & Match & Mismatch & $\begin{array}{l}\text { Match } \\
\text { effect }\end{array}$ \\
\hline Preceding & Match & 0.71 & 2.67 & 1.96 & 0.49 & 7.68 & 7.19 & 4.09 & 2.42 & -1.67 & 2.61 & 4.38 & 1.77 \\
\hline trial: & Mismatch & $\begin{array}{l}0.45 \\
0.18\end{array}$ & 2.23 & 1.78 & $\begin{array}{l}0.53 \\
4.35\end{array}$ & 3.37 & 2.84 & $\begin{array}{l}2.71 \\
-0.47\end{array}$ & 1.51 & -1.20 & $\begin{array}{l}2.88 \\
-0.30\end{array}$ & 5.05 & 2.17 \\
\hline Experiment $1 \mathrm{~b}$ & (reading) & & & & & & & & & & & & \\
\hline Current trial: & & $\begin{array}{r}\text { Relevar } \\
\text { valid }\end{array}$ & $\begin{array}{l}\text { nt dimension } \\
\text { dity }\end{array}$ & low & $\begin{array}{r}\text { Relevar } \\
\text { valid }\end{array}$ & $\begin{array}{l}\text { ht dimension } \\
\text { lity }\end{array}$ & high & $\begin{array}{r}\text { Irreleva } \\
\text { valid }\end{array}$ & $\begin{array}{l}\text { Int dimensior } \\
\text { lity }\end{array}$ & on low & $\begin{array}{r}\text { Irreleva } \\
\text { valid }\end{array}$ & $\begin{array}{l}\text { ant dimensiol } \\
\text { dity }\end{array}$ & n high \\
\hline & & Match & Mismatch & $\begin{array}{l}\text { Match } \\
\text { effect }\end{array}$ & Match & Mismatch & $\begin{array}{l}\text { Match } \\
\text { effect }\end{array}$ & Match & Mismatch & $\begin{array}{l}\text { Match } \\
\text { effect }\end{array}$ & Match & Mismatch & $\begin{array}{l}\text { Match } \\
\text { effect }\end{array}$ \\
\hline Preceding & Match & 1.34 & 2.47 & 1.13 & 1.56 & 4.63 & 3.07 & 2.00 & 2.73 & 0.73 & 2.53 & 2.22 & -0.31 \\
\hline trial: & Mismatch & 1.98 & 1.70 & -0.18 & 1.34 & 3.80 & 2.46 & 2.65 & 2.36 & -0.29 & 2.52 & 1.97 & -0.55 \\
\hline $\begin{array}{l}\text { Adaption effect } \\
\text { Experiment } 2(\mathrm{p}\end{array}$ & predict) & 1.31 & & & 0.61 & & & 1.02 & & & 0.24 & & \\
\hline Current trial: & & Match & Mismatch & $\begin{array}{l}\text { Match } \\
\text { effect }\end{array}$ & & & & & & & & & \\
\hline Preceding & Match & 0.58 & 2.68 & 2.10 & & & & & & & & & \\
\hline trial: & Mismatch & 1.29 & 2.07 & 0.78 & & & & & & & & & \\
\hline Adaption effect & & 1.32 & & & & & & & & & & & \\
\hline
\end{tabular}

Mean response times in ms (a) and \% errors (b) of the different subject groups (per experiment) for match and mismatch trials with respect to match or mismatch in the preceding trial. The match effect is the (usually positive) difference between current match and current mismatch. The adaptation effect marks the reduction of the match effect in succession of a mismatch trial. Numbers are bold if the interaction of match and previous match proved to be statistically significant in the specific sample.

match and a large cost in case of a mismatch could be expected). The impact of preparation on the entire RT distribution could explain why our main RT analyses based on medians per participant and condition (chosen as a robust estimate) led to the same ANOVA results in the current and the other experiments of this study as would be obtained with means per condition. ${ }^{1} \quad$ Table 1

\footnotetext{
${ }^{1}$ We thank Yoav Kessler for directing our attention to this issue.
}

\section{References}

Abrahamse, E. L., Jiménez, J., Verwey, W. B., \& Clegg, B. A. (2010). Representing serial action and perception. Psychonomic Bulletin \& Review, 17, 603-623.

Acosta, E. (1982). Subjective and objective determinants of expectancy: Similarities and differences. The American Journal of Psychology, 95, 139-160.

Alpay, G., Goerke, M., \& Stürmer, B. (2009). Precueing imminent conflict does not override sequence-dependent interference adaptation. Psychological Research, 73, 803-816.

Bubic, A., von Cramon, D. Y., \& Schubotz, R. I. (2010). Prediction, cognition, and the brain. Frontiers in Human Neuroscience, 4, 1-15. 
Dale, R., Duran, N. D., \& Morehead, J. R. (2012). Prediction during statistical learning, and implications for the implicit/explicit divide. Advances in Cognitive Psychology, 8(2), 196-209.

Donkin, C., Brown, S. D., \& Heathcote, A. (2009). ChoiceKey: A realtime speech recognition program for psychology experiments with a small response set. Behavioral Research Methods, 41, 154-162.

Downing, P. E. (2000). Interactions between visual working memory and selective attention. Psychological Science, 11, 467-473.

Duthoo, W., Wühr, P., \& Notebaert, W. (2013). The hot-hand fallacy in cognitive control: Repetition expectancy modulates the congruency sequence effect. Psychonomic Bulletin \& Review, 20, 798-805.

Gaissmaier, W., \& Schooler, L. J. (2008). The smart potential behind probability matching. Cognition, 109, 416-422.

Gaschler, R., \& Nattkemper, D. (2012). Instructed task demands and utilization of action effect anticipation. Frontiers in Psychology, 3, 578

Gaschler, R., Schwager, S., Umbach, V. J., Frensch, P. A., \& Schubert, T. (2014). Expectation mismatch: Differences between self-generated and cue-induced expectations. Neuroscience and Biobehavioral Reviews, 46, 139-157.

Gratton, G., Coles, M. G. H., \& Donchin, E. (1992). Optimizing the use of information: Strategic control of activation of responses. Journal of Experimental Psychology: General, 121, 480-506.

Gray, W. D., Sims, C. R., Fu, W.-T., \& Schoelles, M. J. (2006). The soft constraints hypothesis: A rational analysis approach to resource allocation for interactive behavior. Psychological Review, 113(3), 461-482.

Hacker, M. J., \& Hinrichs, J. V. (1974). Multiple predictions in choice reaction time: A serial memory scanning interpretation. Journal of Experimental Psychology, 103, 999-1005.

Hacker, M. J., \& Hinrichs, J. V. (1979). Verbal predictions of unexpected stimuli and choice reaction time. Memory \& Cognition, 7, 505-510.

Heuer, A., \& Schubö, A. (2016). The focus of attention in visual working memory: Protection of focused representations and its individual variation. PloS One, 11(4), e0154228.

Jiménez, L., \& Méndez, A. (2013). It is not what you expect: Dissociating conflict adaptation from expectancies in a Stroop task. Journal of Experimental Psychology: Human Perception and Performance, 39, 271-284.

Kemper, M., Gaschler, R., Schwager, S., \& Schubert, T. (2016). The benefit of expecting no conflict-Stronger influence of selfgenerated than cue-induced conflict expectations on Stroop performance. Acta Psychologica, 163, 135-141.

Kemper, M., Umbach, V. J., Schwager, S., Gaschler, R., Frensch, P. A., \& Stürmer, B. (2012). Stronger effects of self-generated vs. cueinduced expectations in event-related potentials. Frontiers in Psychology, 3, 562. doi:10.3389/fpsyg.2012.00562

LaRocque, J. J., Lewis-Peacock, J. A., Drysdale, A. T., Oberauer, K., \& Postle, B. R. (2013). Decoding attended information in short-term memory: An EEG Study. Journal of Cognitive Neuroscience, 25, 127-142.

Lewis-Peacock, J. A., Drysdale, A. T., Oberauer, K., \& Postle, B. R. (2012). Neural evidence for a distinction between short-term memory and the focus of attention. Journal of Cognitive Neuroscience, 24, 61-79.

Lindsen, J. P., \& de Jong, R. (2010). Distinguishing between the partialmapping preparation hypothesis and the failure-to-engage hypothesis of residual switch costs. Journal of Experimental Psychology: Human Perception and Performance, 36, 1207-1226.

Marcus, D. J., Karatekin, C., \& Markiewicz, S. (2006). Oculomotor evidence of sequence learning on the serial reaction time task. Memory \& Cognition, 34, 420-432.

Mattler, U. (2005). Combined expectancy effects: An accumulator model. Cognitive Psychology, 51, 214-255.

Mattler, U., van der Lugt, A., \& Münte, T. F. (2006). Combined expectancies: Electrophysiological evidence for adjusted expectancy effects. BMC Neuroscience, 7, 37.

Meiran, N. (1996). Reconfiguration of processing mode prior to task performance. Cognition, 22, 1423-1442.

Memelink, J., \& Hommel, B. (2013). Intentional weighting: A basic principle in cognitive control. Psychological Research, 77, 249259.

Mordkoff, J., \& Halterman, R. (2008). Feature integration without visual attention: Evidence from the correlated flankers task. Psychonomic Bulletin \& Review, 15(2), 385-389.

Oberauer, K., Souza, A. S., Druey, M. D., \& Gade, M. (2013). Analogous mechanisms of selection and updating in declarative and procedural working memory: Experiments and a computational model. Cognitive Psychology, 66, 157-211.

Pan, Y. (2010). Attentional capture by working memory contents. Canadian Journal of Experimental Psychology, 64(2), 124-128.

Pfister, R., Kiesel, A., \& Hoffmann, J. (2011). Learning at any rate: Action-effect learning for stimulus-based actions. Psychological Research, 75, 61-65.

Pfister, R., Kiesel, A., \& Melcher, T. (2010). Adaptive control of ideomotor effect anticipations. Acta Psychologica, 135, 316-322.

Rogers, R., \& Monsell, S. (1995). Costs of a predictable switch between simple cognitive tasks. Journal of Experimental Psychology: General, 124, 207-231.

Schwager, S., \& Hagendorf, H. (2009). Goal-directed access to mental objects inworking memory: The role of task-specific feature retrieval. Memory \& Cognition, 37, 1103-1119.

Umbach, V. J., Schwager, S., Frensch, P. A., \& Gaschler, R. (2012). Does explicit expectation really affect preparation? Frontiers in Psychology, 3, 378.

Wolfensteller, U., \& Ruge, H. (2014). Response selection difficulty modulates the behavioral impact of rapidly learnt action effects. Frontiers in Psychology, 5, 1382. 